\title{
Pathological observations of lung inflammation after administration of IP-10 in influenza virus- and respiratory syncytial virus-infected mice
}

\author{
HONG LUO ${ }^{1,2}$, DONG WANG ${ }^{3}$, HAI-LONG CHE ${ }^{1}$, YUE ZHAO ${ }^{4}$ and HONG JIN ${ }^{1}$ \\ ${ }^{1}$ Department of Microbiology and Parasitology, China Medical University, Shenyang 110001; \\ ${ }^{2}$ Department of Clinical Microbiology, Dalian Medical University, Dalian 116044; ${ }^{3}$ Maternal and Child Health Center \\ of Langfang City, Langfang 065000; ${ }^{4}$ Department of Pathology, China Medical University, Shenyang 110001, P.R. China
}

Received May 27, 2011; Accepted July 12, 2011

DOI: $10.3892 / \mathrm{etm} .2011 .350$

\begin{abstract}
Pneumonia is a common complication of influenza virus infection and a common cause of death of patients. The aim of this study was to test the hypothesis that interferon- $\gamma$ inducible protein-10 (IP-10) is an important chemokine in the development of airway inflammation caused by certain viruses. Mice were infected with influenza virus after administration of murine IP-10 and the severity of pneumonia was compared with the group which was infected with influenza virus alone. Another mice group was infected with respiratory syncytial virus (RSV) after injection of IP-10 and also the severity of pneumonia was compared to the group which was infected with RSV alone. The mice infected with influenza virus or RSV after administration of IP-10 presented with more fulminant and necrotizing diffuse alveolar and bronchiole damage with lymphocyte infiltration. Our results indicate that IP-10 is an important chemokine and is associated with the severity of pneumonia caused by certain viruses.
\end{abstract}

\section{Introduction}

Influenza has been responsible for millions of deaths worldwide. Influenza virus type A is antigenically highly variable and is responsible for most cases of epidemic influenza. In 1997, the first instance of human respiratory disease and death associated with the H5N1 avian influenza virus was documented. In 2009, the outbreak of human infection with a novel influenza A (H1N1) virus, which spread through sustained human-to-human transmission, caused a world pandemic.

When a person is infected with uncomplicated influenza, the symptoms usually last for a short period and the patient

Correspondence to: Dr Hong Jin, Department of Microbiology and Parasitology, China Medical University, No. 92 Bei Er Road, Heping, Shenyang 110001, P.R. China

E-mail: hong-jin@excite.com

Key words: interferon- $\gamma$-inducible protein-10, pneumonia, influenza virus, respiratory syncytial virus recovers rapidly, but serious complications may occur in elderly individuals and those with underlying chronic diseases. Pneumonia is a common complication of influenza virus infection. Primary viral pneumonia is a major manifestation of human H5N1 disease, and is a common cause of death of patients with influenza virus infection (1). Yet, the biological basis for the severity of human H5N1 disease and fatal cases of the novel influenza $\mathrm{A}(\mathrm{H} 1 \mathrm{~N} 1)$ virus infection remains unclear.

High levels of interferon- $\gamma$-inducible protein-10 (IP-10) have been observed in several viral diseases $(2,3)$. Infection of $\mathrm{H} 5 \mathrm{~N} 1$ virus was found to lead to the hyper-induction of proinflammatory cytokines in human primary macrophage cultures in vitro, and patients with $\mathrm{H} 5 \mathrm{~N} 1$ disease were found to have unusually high serum concentrations of chemokine IP-10 (4). The hyper-induction of chemokines may contribute to the severity of human $\mathrm{H} 5 \mathrm{~N} 1$ disease and this may be relevant to the pathogenesis of pneumonia caused by influenza virus.

To test the hypothesis whether IP-10 is an important chemokine in the development of airway inflammation caused by virus, mice were infected with influenza virus after administration of murine IP-10, and the severity of pneumonia was compared to that of the mouse group infected with influenza virus alone. Another mouse group was infected with respiratory syncytial virus (RSV) after being injected with murine IP-10, and also the inflammatory response of the lung was compared to that of the mouse group infected with RSV alone.

\section{Materials and methods}

Mice. Pathogen-free 8-week-old female BALB/c mice were purchased from the Animal Center of China Medical University. Mice were bred and/or housed under specific, pathogen-free conditions until the day of sacrifice. The institutional ethics committee for animal use and care approved all animal-related experiments and procedures.

IP-10 administration to mice. Murine recombinant IP-10 was purchased from Shanghai BioSun Sci \& Tech Co., Ltd. Mice received intraperitoneal (i.p.) injections of $30 \mu \mathrm{g} / \mathrm{kg}$ IP-10 in $0.2 \mathrm{ml}$ of phosphate-buffered saline (PBS; Gibco/BRL) on day 0-2 of virus infection. 
Viral infection. Mouse-adapted influenza A/FM/1/47 H1N1 viruses were cultivated in the allantoic cavity of 10-day-old chicken embryos by incubating infected embryos at $34^{\circ} \mathrm{C}$ for 2-3 days. RSV was propagated in HeLa cells.

Under light anesthesia, mice were inoculated intranasally (i.n.) with $50 \mu \mathrm{l}$ of 1:10 diluted influenza virus stock containing $10^{4} 50 \%$ egg lethal dose (ELD50) per milliliter of allantoic fluid or $10^{7}$ plaque forming units (PFUs) of RSV in a volume of $50 \mu \mathrm{l}$. For influenza virus-infected mice, Group 1 was infected with influenza virus without administration of IP-10; Group 2 was infected with influenza virus after administration of IP-10; Group 3 was mock-infected with PBS; and Group 4 was injected with IP-10 without virus infection. For RSV-infected mice, Group 1 was infected with RSV alone and Group 2 was infected with RSV after administration of IP-10; Group 3 was mock-infected. At 5 days post-infection, mice were euthanized. The number of mice per group was 10 .

Measurement of lung weight. The mice were sacrificed by cervical dislocation. Lungs were removed, and the lung index of lungs, i.e., lung weight/body weight x 100, was determined as an indication of lung inflammation, as previously described (5).

Pulmonary histopathology. The left lobe of the lung was perfused, fixed in $10 \%$ buffered formalin and embedded in paraffin. Mutiple sections $(4-\mu \mathrm{m})$ were stained with H\&E. Slides were analyzed and scored for cellular inflammation under light microscopy by two independent pathologists, as previously described (6). Inflammatory infiltrates were scored by enumerating the layers of inflammatory cells surrounding the vessels, bronchioles and alveolar infiltration. An overall severity score was calculated for each animal by adding the individual scores. Histopathology scores for animal from two independent experiments were pooled and averaged, and the standard error of the mean (SEM) was calculated. Lung histopathological results of the virus infection group and IP-10 administration/virus infection group were compared using the Mann-Whitney U test. Differences were considered significant at a $\mathrm{P}$-value $<0.05$.

\section{Results}

Lung index. Fig. 1 shows the lung index in the different groups of mice. The extent of inflammation was evaluated by the increase in lung weight using the lung index. There was a significant increase in the lung index in Group 2 mice which were infected with the influenza virus after administration of IP-10 (Fig. 1A) and in Group 2 mice infected with RSV after administration of IP-10 (Fig. 1B).

Lung inflammation in the influenza virus-infected mice. A well-known function of chemokines is to drive leukocyte migration through tissues to target microenvironments. Therefore, the histological appearance of the lungs and the degree of inflammation were investigated. Group 2 (8-weekold female BALB/c mice) was infected with influenza virus after administration of murine IP-10 and the result was compared with Group 1 mice which were infected with influenza virus without IP-10 administration and the mock-infected Group 3. On day 5 post-infection, multiple H\&E-stained lung
A

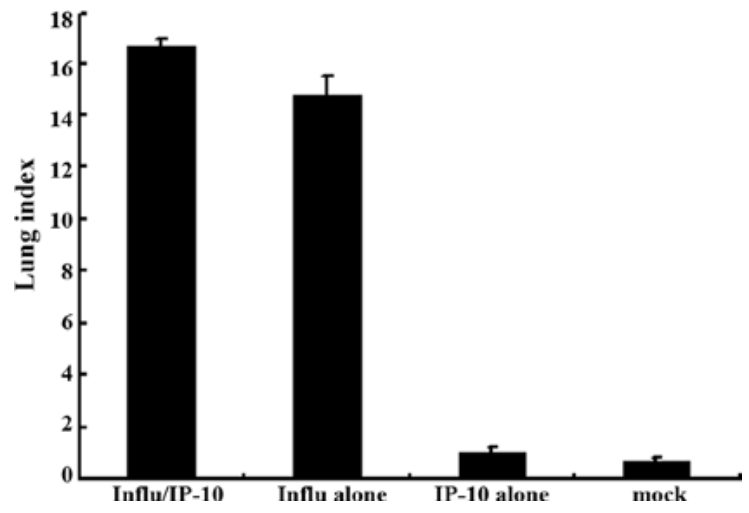

B

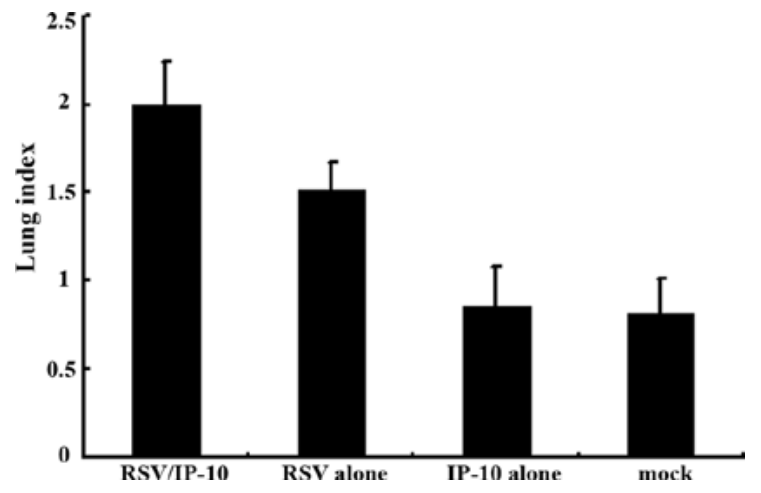

Figure 1. Lung index, i.e., lung weight/body weight $\mathrm{x} 100$, of the lungs of mice injected with IP-10 after infection with (A) influenza virus or (B) RSV.

sections were analyzed and inflammation was scored using a previously described scoring scale (6).

In the mock-infected mice (Fig. 2A) and Group 4 which was injected with IP-10 alone (data not shown), few infiltrating cells were found in interstitial/alveolar ducts and around bronchioles or vessels. The mice in Group 2 which were infected with influenza virus after administration of IP-10, had a greater degree of interstitial and alveolar inflammation (Fig. 2C) compared to the mice in Group 1 (Fig. 2B) which were infected with influenza virus alone. The pulmonary pathology of Group 2 was acute exudative diffuse alveolar damage with hyaline membrane formation and the major cell types of infiltration were lymphocytes and, to a lesser extent, macrophages. The peribronchiolar inflammation in Group 2 was also much more severe compared to Group 1, with diffuse damage of structures.

The overall severity scores differed significantly $(\mathrm{P}<0.05)$ between the two groups (Groups 1 and 2), and the overall severity score indicated a trend toward a greater magnitude in Group 2 (Fig. 4A).

Lung inflammation in RSV-infected mice. To survey the pulmonary inflammation of mice infected with RSV after administration of murine IP-10, the histological appearance of the lung and the degree of inflammation of RSV-infected mice were investigated. The mice were also divided into three groups similar to the influenze-infected mice. The RSV-infected and IP-10-injected mice (Group 2) were compared to Group 1 mice which were infected with RSV without IP-10 administration and mock-infected Group 3. On day 5 post-infection, multiple $\mathrm{H} \& \mathrm{E}$-stained lung sections were analyzed, and inflammation was scored using a scoring scale as described above. 

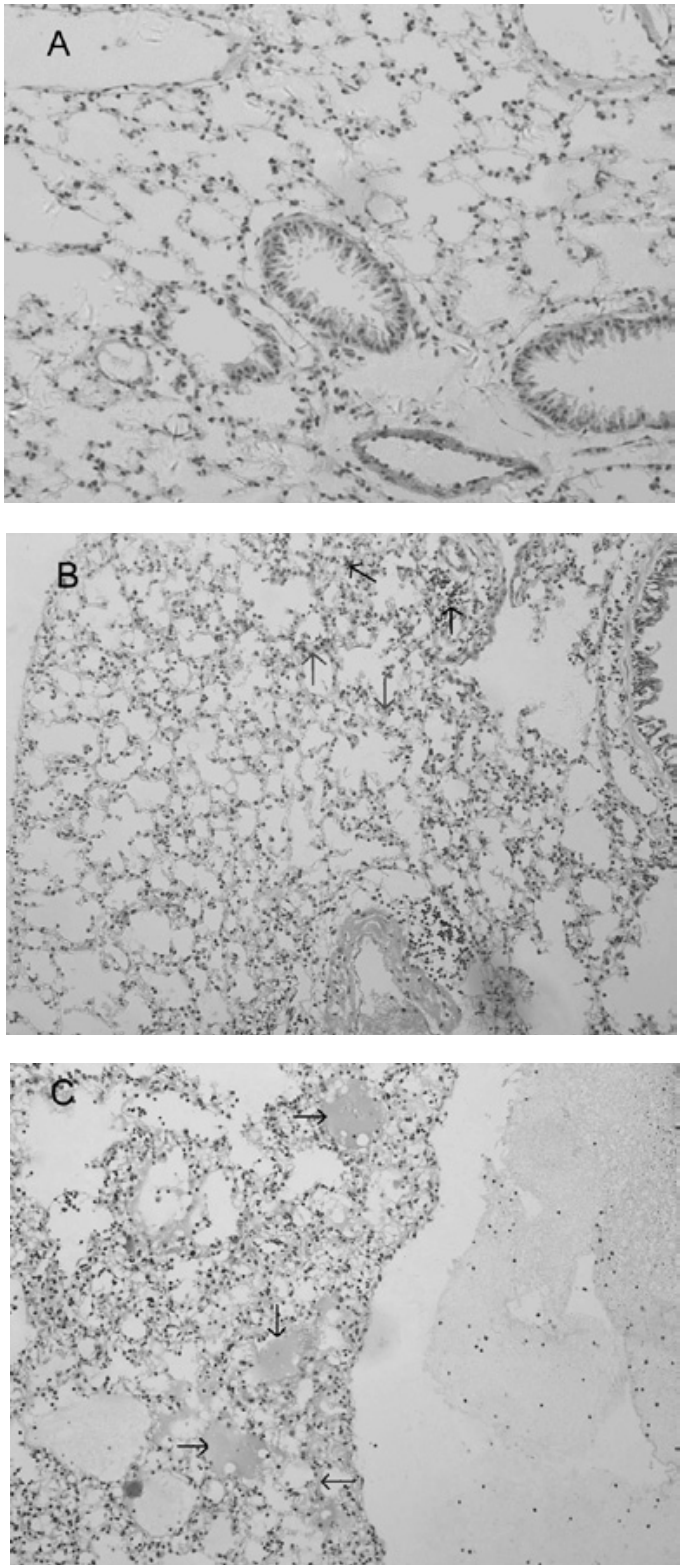

Figure 2. Lung histopathology of BALB/c mice infected with influenza virus. On day 5 post-infection, mice were sacrificed. Lungs were removed, fixed in $10 \%$ buffered formalin and embedded in paraffin. Multiple sections $(4-\mu \mathrm{m})$ were stained with $\mathrm{H} \& \mathrm{E}$. Original magnification, x200. Mice were (A) mockinfected; (B) infected i.n. with $10^{4}$ ELD50 influenza virus which showed interstitial lymphocyte infiltration (arrows); or (C) infected with influenza virus after administration of IP-10 which showed acute exudative alveolar damage with hyaline membrane (arrows).

Group 2 mice which were infected with RSV virus after administration of IP-10 had a greater degree of interstitial and alveolar inflammation (Fig. 3C) compared to Group 1 mice which were infected with RSV alone (Fig. 3B) and mock-infected mice (Fig. 3A). The pulmonary pathology of Group 2 was acute exudative diffuse alveolar damage, but no hyaline membrane formation was noted, and the major cell types involved in infiltration were lymphocytes which was the same as the influenza virus-infected group; the peribronchiolar inflammation in this group was also much more severe compared to Group 1, with damage of structures.

The overall severity scores differed significantly $(\mathrm{P}<0.05)$ between the two groups (Groups 1 and 2) (Fig. 4B).
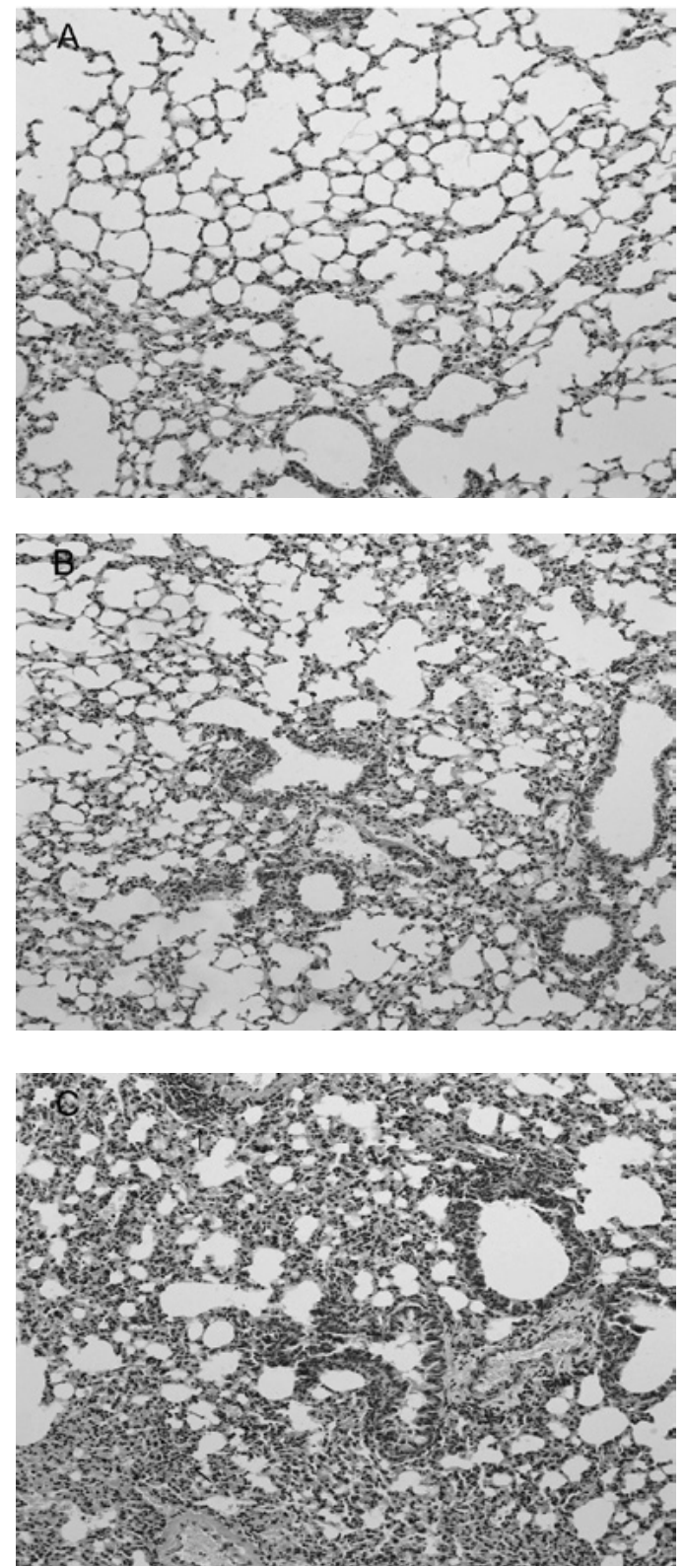

Figure 3. Lung histopathology of BALB/c mice infected with RSV. On day 5 post-infection, mice were sacrificed. Lungs were removed, fixed in $10 \%$ buffered formalin and embedded in paraffin. Multiple sections $(4-\mu \mathrm{m})$ were stained with H\&E. Original magnification, x200. Mice were (A) mock-infected; (B) infected i.n. with $10^{7} \mathrm{PFU}$ RSV or (C) with RSV after administration of IP-10, which had a greater degree of interstitial lymphocyte infiltration (arrows).

\section{Discussion}

Patients with H5N1 disease may present with primary viral pneumonia complicated by syndromes of acute respiratory distress and multiple organ dysfunction. Findings in the respiratory tree consist of diffuse alveolar damage with interstitial lymphoplasmacytic infiltrate and the formation of hyaline membranes in alveoli/alveolar ducts in addition to necrotizing bronchitis and bronchiolitis. In cases of coincident bacterial pneumonia, a massive infiltration of neutrophils into alveolar air spaces is observed. The interstitial pneumonia caused by the $\mathrm{H} 5 \mathrm{~N} 1$ virus is much more severe compared to the pneumonia caused by other types of influenza viruses (7). In the present 
A
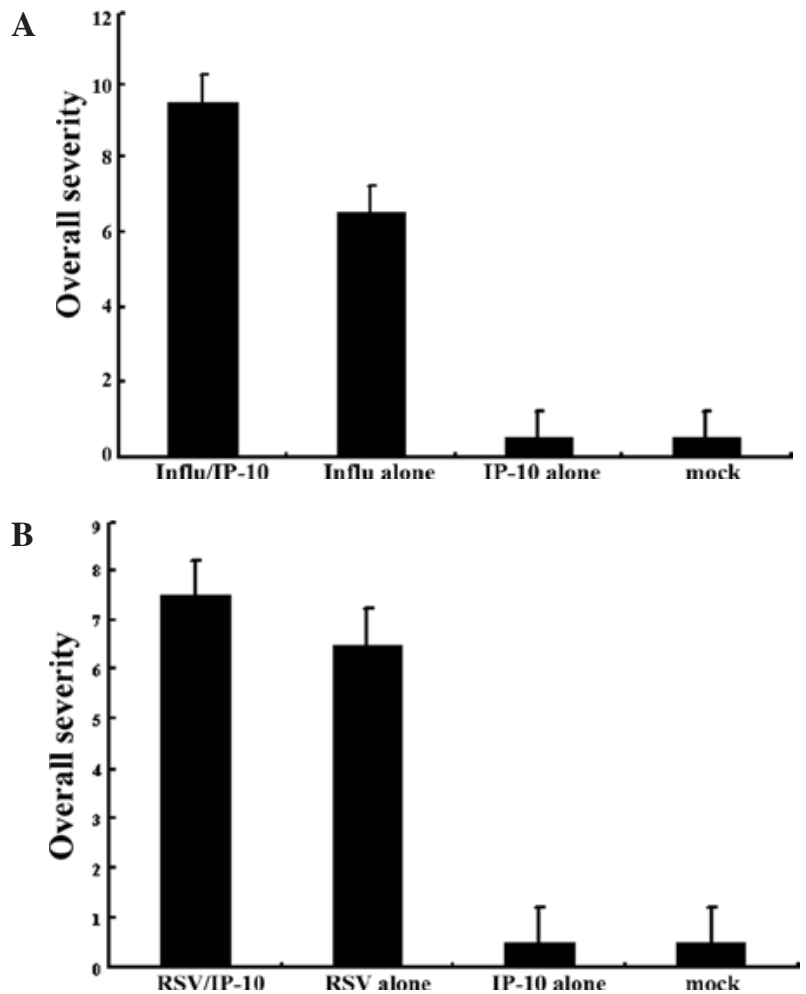

Figure 4. Inflammation scores from the lung histopathology sections as determined after infection with the (A) influenza virus or (B) RSV.

study, intranasal administration of influenza virus to mice led to the development of pneumonia, characterized by alveolar damage with lymphoplasmacytic infiltration and interstitial edema. A pseudostratified change in columnar epithelial cells of bronchitis and bronchiolitis occurred. The mock-infected group had no interstitial and alveolar inflammation.

Chemokines are a large family of proteins that mediate the movement and activation of diverse groups of inflammatory cells. A subfamily of chemokines, including IP-10, plays a key role in promoting type I immune responses. IP-10 contributes to lung inflammatory cell recruitment and activation which directs CD8(+) T-cell recruitment to the lungs (8). IP-10 is an important factor in the clearance of certain pathogens, but it also causes severe inflammatory reaction in lungs and may be associated with pulmonary pathological changes that cause acute respiratory distress in patient. Dawson et al (9) found IP-10 mRNA overexpression in the lungs after influenza virus infection. Patients with H5N1 disease have unusually high serum concentrations of chemokine IP-10, and the hyperinduction of this chemokine may contribute to the unusual severity of human H5N1 disease (10). The results of the present study showed that the mice infected with influenza virus after administration of IP-10 suffered a more fulminant and necrotizing diffuse alveolar and bronchiole damage with acute exudative inflammation as well as hyaline membrane formation and strongly reactive lymphocytes; the result also showed that the pneumonia of this group was more severe compared to the group of mice infected with influenza virus alone. The overall severity scores were significantly higher for the group of IP-10-injected mice. This result indicates that IP-10 plays an important role in the development of severe pneumonia.
RSV is the most important cause of lower respiratory tract illness in infants and children. The group of mice infected with RSV after administration of IP-10 demonstrated a greater degree of interstitial and alveolar inflammation than the group of mice infected with RSV without IP-10 administration. These results suggest that IP-10 is also associated with the severity of pneumonia caused by RSV. However, RSV-induced pneumonia had less interstitial and alveolar inflammation than that of the influenza virus-infected group (the overall severity scores differed significantly between the two groups; $\mathrm{P}<0.05)$. This indicates that IP-10 may have a much more important correlation with the severity of influenza virus-induced pneumonia. Zeng et al (11) showed that IP-10 was an important component of innate immunity against extracellular bacterial pathogens of the lung. Nevertheless, in certain viral pneumonias the level of IP-10 was significantly elevated and produced immunopathological processes involved in lung injury (12). In conclusion, IP-10 is an important chemokine and is associated with the severity of pneumonia caused by several types of viruses and may be used as a candidate for immunoadjuvant therapy in the future.

\section{References}

1. Cheng CY, Poon LLM, Lau AS, Luk W, Lau YL, Shortridge KF, Guan Y and Peiris JSM: Induction of proinflammatory cytokines in human macrophages by influenza A (H5N1) viruses: a mechanism for the unusual severity of human disease? Lancet 360: 1831-1837, 2002.

2. Antonelli A, Ferri C, Fallahi P, Ferrari SM, Sebastiani M, Ferrari D, Giunti M, Frascerra S, Tolari S, Franzoni F, Galetta F, Marchi S and Ferrannini E: High values of CXCL10 serum levels in mixed cryoglobulinemia associated with hepatitis $\mathrm{C}$ infection. Am J Gastroenterol 103: 2488-2494, 2008.

3. Bhowmick S, Duseja R, Das S, Appaiahgiri MB, Vrati S and Basu A: Induction of IP-10 (CXCL10) in astrocytes following Japanese encephalitis. Neurosci Lett 414: 45-50, 2007.

4. Chan MCW, Cheung CY, Chui WH, Tsao SW, Nicholls JM, Chan YO, Chan RWY, Long HT, Poon LLM, Guan Y and Peiris JSM: Proinflammatory cytokine responses induced by influenza A (H5N1) viruses in primary human alveolar and bronchial epithelial cells. Respir Res 6: 135-147, 2005.

5. Allen EM, Moore VL and Stevens JO: Strain variation in BCG-induced chronic pulmonary inflammation in mice. I. Basic model and possible genetic control by non-H-2 genes. J Immunol 119: 343-347, 1977.

6. Kajon AE, Gigliotti AP and Harrod KS: Acute inflammatory response and remodeling of airway epithelium after subspecies B1 human adenovirus infection of the mouse lower respiratory tract. J Med Virol 71: 233-244, 2003.

7. Jeffery KT and David MM: The pathology of influenza virus infection. Ann Rev Pathol 3: 499-522, 2008.

8. Zeng X, Moore TA, Newstead MW, Deng JC, Lukacs NW and Standiford TJ: IP-10 mediates selective mononuclear cell accumulation and activation in response to intrapulmonary transgenic expression and during adenovirus-induced pulmonary inflammation. J Interferon Cytokine Res 25: 103-112, 2005.

9. Dawson TC, Beck MA, Kuziel WA, Henderson F and Maeda N: Contrasting effects of CCR5 and CCR2 deficiency in the pulmonary inflammatory response to influenza A virus. Am J Pathol 156: 1951-1959, 2000.

10. Peiris JSM, Yu WC, Leung CW, Cheung CY, Ng WF, Nicholls JM, Ng TK, Chan KH, Lai ST, Lim WL, Yuen KY and Guan Y: Re-emergence of fatal human influenza A subtype H5N1 disease. Lancet 363: 617-619, 2004.

11. Zeng X, Moore TA, Newstead MW, Deng JC, Kunkel SL, Luster AD and Standiford TJ: Interferon-inducible protein 10, but not monokine induced by gamma interferon, promotes protective type 1 immunity in murine klebsiella pneumoniae pneumonia. Infect Immun 73: 8226-8236, 2005.

12. Chien JY, Hsueh PR, Cheng WC, Yu CJ and Yang PC: Temporal changes in cytokine/chemokine profiles and pulmonary involvement in severe acute respiratory syndrome. Respirology 11: 715-722, 2006 . 